\title{
The Effect of Satellite Observing System Changes on MERRA Water and Energy Fluxes
}

Franklin R. Robertson ${ }^{1}$, M. G Bosilovich ${ }^{2}$, J. Chen ${ }^{2,3}$, and T. L. Miller ${ }^{1}$

${ }^{1}$ NASA / MSFC

${ }^{2}$ NASA / GSFC/GMAO

${ }^{3} \mathrm{UMD} / \mathrm{ESSIC}$

Submitted to AGU Fall 2010 Session A20: Progress and Uncertainty in Reanalysis Datasets

Because reanalysis data sets offer state variables and fluxes at regular space / time intervals, atmospheric reanalyses have become a mainstay of the climate community for diagnostic purposes and for driving offline ocean and land models. Although one weakness of these data sets is the susceptibility of the flux products to uncertainties because of shortcomings in parameterized model physics, another issue, perhaps less appreciated, is the fact that continual but discreet changes in the evolving observational system, particularly from satellite sensors, may also introduce artifacts in the time series of quantities.

In this paper we examine the ability of the NASA MERRA (Modern Era Retrospective Analysis for Research and Applications) and other recent reanalyses to determine variability in the climate system over the satellite record ( the last 30 years). In particular we highlight the effect on the reanalysis of discontinuities at the junctures of the onset of passive microwave imaging (Special Sensor Microwave Imager) in late 1987 as well as improved sounding and imaging with the Advanced Microwave Sounding Unit, AMSU-A, in 1998. We first examine MERRA fluxes from the perspective of how physical modes of variability (e.g. ENSO events, Pacific Decadal Variability) are contamined by artificial step-like trends induced by the onset of new moisture data these two satellite observing systems. Secondly, we show how Redundancy Analysis, a statistical regression methodology, is effective in relating these artifact signals in the moisture and temperature analysis increments to their presence in the physical flux terms (e.g. precipitation, radiation). This procedure is shown to be effective greatly reducing the artificial trends in the flux quantities. 